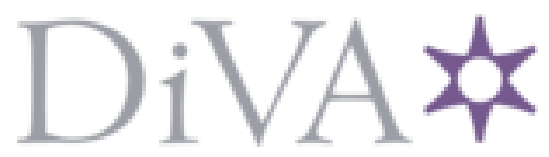

http://www.diva-portal.org

This is the published version of a paper published in Physics of Plasmas.

Citation for the original published paper (version of record):

Brodin, G., Stenflo, L. (2014)

Nonlinear dynamics of large amplitude modes in a magnetized plasma.

Physics of Plasmas, 21(12)

http://dx.doi.org/10.1063/1.4903326

Access to the published version may require subscription.

N.B. When citing this work, cite the original published paper.

Permanent link to this version:

http://urn.kb.se/resolve?urn=urn:nbn:se:umu:diva- 104152 


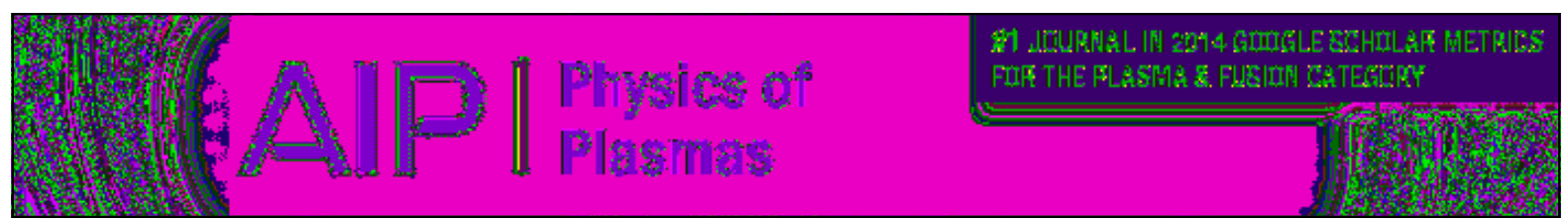

\section{Nonlinear dynamics of large amplitude modes in a magnetized plasma}

G. Brodin and L. Stenflo

Citation: Physics of Plasmas 21, 122301 (2014); doi: 10.1063/1.4903326

View online: http://dx.doi.org/10.1063/1.4903326

View Table of Contents: http://scitation.aip.org/content/aip/journal/pop/21/12?ver=pdfcov

Published by the AIP Publishing

\section{Articles you may be interested in}

Study of nonlinear dynamics in magnetron by using circuitry model

Phys. Plasmas 19, 032312 (2012); 10.1063/1.3694843

Transit time instabilities in an inverted fireball. II. Mode jumping and nonlinearities

Phys. Plasmas 18, 012105 (2011); 10.1063/1.3533440

Nonlinear transformation of electromagnetic wave in time-varying plasma medium: Longitudinal propagation J. Appl. Phys. 100, 023303 (2006); 10.1063/1.2218035

Adiabatic bistable evolution of dynamical systems governed by a Hamiltonian with separatrix crossing

Phys. Plasmas 13, 054502 (2006); 10.1063/1.2201927

Nonlinear oscillations in dusty plasmas in the presence of nonisothermal trapped electrons Phys. Plasmas 10, 631 (2003); 10.1063/1.1543577

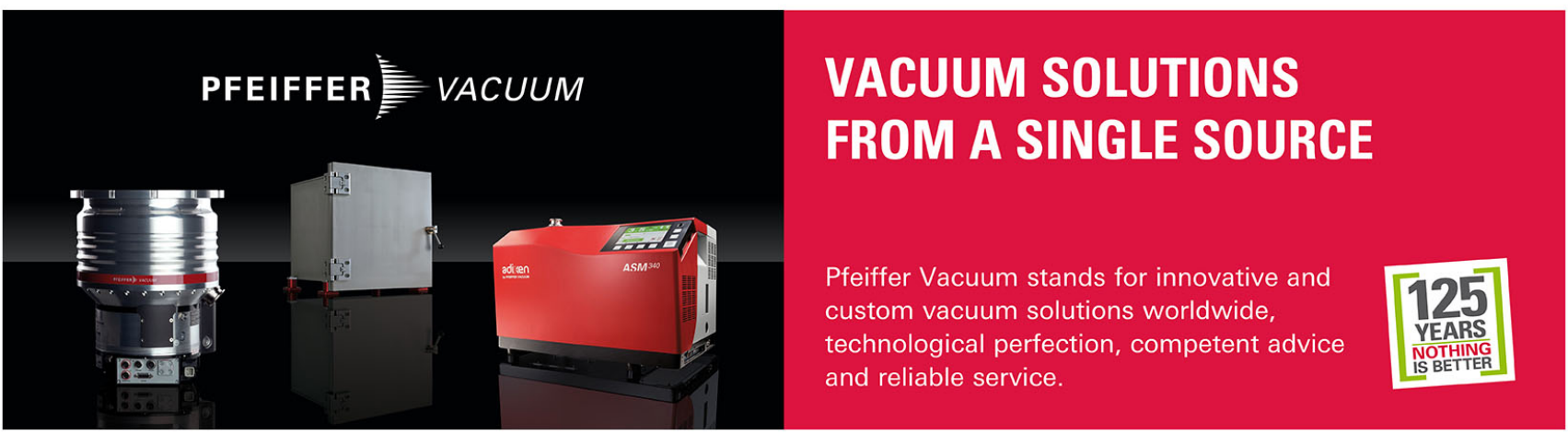




\section{Nonlinear dynamics of large amplitude modes in a magnetized plasma}

G. Brodin ${ }^{1, a)}$ and L. Stenflo ${ }^{2}$

${ }^{1}$ Department of Physics, Umea University, SE-901 87 Umeå, Sweden

${ }^{2}$ Department of Physics, Linköping University, SE-581 83 Linköping, Sweden

(Received 6 November 2014; accepted 17 November 2014; published online 3 December 2014)

We derive two equations describing the coupling between electromagnetic and electrostatic oscillations in one-dimensional geometry in a magnetized cold and non-relativistic plasma. The nonlinear interaction between the wave modes is studied numerically. The effects of the external magnetic field strength and the initial electromagnetic polarization are of particular interest here. New results can, thus, be identified. (C) 2014 AIP Publishing LLC.

[http://dx.doi.org/10.1063/1.4903326]

\section{INTRODUCTION}

Large amplitude electron plasma oscillations are in most cases treated by means of perturbation methods. However, there are a few particular cases for which exact analytical solutions can be found, e.g., Ref. 1-5. In such schemes, one first makes an Ansatz, often by trial and error, on the spatial behavior of the physical variables, such that the partial differential equations (PDE:s) reduce to a system of coupled nonlinear ordinary differential equations for the temporal evolutions of the wave amplitudes. Such systems (see also Refs. 6-8) can be very useful, in particular, in comparisons with more general, although approximate, PDE:s derived by other techniques.

Recently, we considered wave propagation in a cold plasma. ${ }^{9}$ In that case, we had, due to mathematical difficulties, to assume that the plasma was unmagnetized in its equilibrium state. In the present paper, we have however been able to consider wave propagation in a magnetized plasma for the case, where the waves propagate in the direction of a constant magnetic field $B_{0} \hat{\mathbf{z}}$. In this way, it has been possible for us to derive a generalized system of coupled ordinary differential equations. The presence of the external magnetic field leads to a richer dynamics of the coupled system, as is shown numerically.

\section{BASIC EQUATIONS AND DERIVATIONS}

Let us start from the basic equations for a cold nonrelativistic electron plasma. We then have

$$
\begin{gathered}
\frac{\partial n}{\partial t}+\nabla \cdot(n \mathbf{v})=0 \\
\left(\frac{\partial}{\partial t}+\mathbf{v} \cdot \boldsymbol{\nabla}\right) \mathbf{v}=-\frac{e}{m}(\mathbf{E}+\mathbf{v} \times \mathbf{B}) \\
\nabla \times \mathbf{E}=-\frac{\partial \mathbf{B}}{\partial t} \\
\nabla \times \mathbf{B}=-e \mu_{0} n \mathbf{v}+\frac{1}{c^{2}} \frac{\partial \mathbf{E}}{\partial t}
\end{gathered}
$$

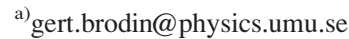

and

$$
\boldsymbol{\nabla} \cdot \mathbf{E}=-\frac{e\left(n-n_{0}\right)}{\varepsilon_{0}}
$$

Here, $n$ is the electron number density, $n_{0}$ is the constant ion number density, $\mathbf{v}$ is the electron fluid velocity, $\mathbf{E}$ is the electric field, $\mathbf{B}$ is the magnetic field, $-e / m$ is the electron charge to mass ratio, $\mu_{0}$ is the magnetic vacuum permeability, and $c$ is the speed of light. Next, we consider one-dimensional spatial variations in the z-direction, i.e., $\boldsymbol{\nabla} \rightarrow \hat{\mathbf{z}} \partial / \partial z$. Furthermore, we make the Ansatz $n=n(t), \mathbf{B}=B_{x}(t) \hat{\mathbf{x}}+B_{y}(t) \hat{\mathbf{y}}+B_{0} \hat{\mathbf{z}}, \mathbf{v}$ $=\left(u_{x}(t) \hat{\mathbf{x}}+u_{y}(t) \hat{\mathbf{y}}+u_{z}(t) \hat{\mathbf{z}}\right) z, \quad$ and $\quad \mathbf{E}=\left(\epsilon_{x}(t) \hat{\mathbf{x}}+\epsilon_{y}(t) \hat{\mathbf{y}}\right.$ $\left.+\epsilon_{z}(t) \hat{\mathbf{z}}\right) z$. Substituting this into Eqs. (1)-(4), we obtain

$$
\begin{gathered}
u_{z}=-\frac{1}{n} \frac{\partial n}{\partial t}, \\
\frac{\partial u_{z}}{\partial t}+u_{z}^{2}=-\frac{e}{m}\left(\epsilon_{z}+u_{x} B_{y}-u_{y} B_{x}\right), \\
\frac{\partial u_{x}}{\partial t}+u_{z} u_{x}=-\frac{e}{m}\left(\epsilon_{x}+u_{y} B_{0}-u_{z} B_{y}\right), \\
\frac{\partial u_{y}}{\partial t}+u_{z} u_{y}=-\frac{e}{m}\left(\epsilon_{y}-u_{x} B_{0}+u_{z} B_{x}\right), \\
\epsilon_{y}=\frac{\partial B_{x}}{\partial t}, \\
u_{x}=\frac{\varepsilon_{0}}{e n} \frac{\partial \epsilon_{x}}{\partial t}=-\frac{\partial B_{y}}{\partial t}, \\
u_{y}=\frac{\varepsilon_{0}}{e n} \frac{\partial \epsilon_{y}}{\partial t}=\frac{\varepsilon_{0}}{e n} \frac{\partial^{2} B_{x}}{\partial t^{2}}
\end{gathered}
$$

and

$$
\epsilon_{z}=-\frac{e\left(n-n_{0}\right)}{\varepsilon_{0}}
$$
yield

Equations (6), (12), and (13) together with (8) and (9) 


$$
\begin{array}{r}
\frac{\partial}{\partial t}\left(-\frac{\varepsilon_{0}}{e n} \frac{\partial^{2} B_{y}}{\partial t^{2}}\right)+\left(-\frac{1}{n} \frac{\partial n}{\partial t}\right)\left(-\frac{\varepsilon_{0}}{e n} \frac{\partial^{2} B_{y}}{\partial t^{2}}\right) \\
=-\frac{e}{m}\left(-\frac{\partial B_{y}}{\partial t}+\frac{\varepsilon_{0}}{e n} \frac{\partial^{2} B_{x}}{\partial t^{2}} B_{0}+\frac{1}{n} \frac{\partial n}{\partial t} B_{y}\right)
\end{array}
$$

and

$$
\begin{aligned}
\frac{\partial}{\partial t} & \left(\frac{\varepsilon_{0}}{e n} \frac{\partial^{2} B_{x}}{\partial t^{2}}\right)+\left(-\frac{1}{n} \frac{\partial n}{\partial t}\right)\left(\frac{\varepsilon_{0}}{e n} \frac{\partial^{2} B_{x}}{\partial t^{2}}\right) \\
& =-\frac{e}{m}\left(\frac{\partial B_{x}}{\partial t}+\frac{\varepsilon_{0}}{e n} \frac{\partial^{2} B_{y}}{\partial t^{2}} B_{0}-\frac{1}{n} \frac{\partial n}{\partial t} B_{x}\right) .
\end{aligned}
$$

Similarly, substituting (6), (12)-(14) into (7) gives

$$
\begin{aligned}
- & \frac{\partial}{\partial t}\left(\frac{1}{n} \frac{\partial n}{\partial t}\right)+\left(\frac{1}{n} \frac{\partial n}{\partial t}\right)^{2} \\
& =\frac{e^{2}}{m}\left[\frac{\left(n-n_{0}\right)}{\varepsilon_{0}}+\frac{\varepsilon_{0}}{n e^{2}}\left(B_{y} \frac{\partial^{2} B_{y}}{\partial t^{2}}+B_{x} \frac{\partial^{2} B_{x}}{\partial t^{2}}\right)\right],
\end{aligned}
$$

where $n_{0}$ is the constant background density.

Next, we introduce normalized variables according to $t \rightarrow \omega_{p} t, \quad n \rightarrow N=n / n_{0}, \quad$ and $\quad B_{x, y} \rightarrow e B_{x, y} / m \omega_{p}$, where $\omega_{p}=\left(n_{0} e^{2} / \varepsilon_{0} m\right)^{1 / 2}$. Moreover, we introduce a complex magnetic field defined by $B_{+}=B_{x}+i B_{y}$. The three Eqs. (15)-(17) can then be rewritten as two coupled equations

$$
\begin{aligned}
- & \frac{\partial}{\partial t}\left(\frac{1}{N} \frac{\partial N}{\partial t}\right)+\left(\frac{1}{N} \frac{\partial N}{\partial t}\right)^{2} \\
& =(N-1)+\frac{\omega_{c}}{N}\left(\operatorname{Im} B_{+} \frac{\partial^{2}\left(\operatorname{Im} B_{+}\right)}{\partial t^{2}}+\operatorname{Re} B_{+} \frac{\partial^{2}\left(\operatorname{Re} B_{+}\right)}{\partial t^{2}}\right)
\end{aligned}
$$

and

$$
\frac{\partial}{\partial t}\left(\frac{1}{N^{2}} \frac{\partial^{2} B_{+}}{\partial t^{2}}\right)+i \frac{\omega_{c}}{N^{2}} \frac{\partial^{2} B_{+}}{\partial t^{2}}=-\frac{\partial}{\partial t}\left(\frac{B_{+}}{N}\right),
$$

where $B_{+}=\operatorname{Re} B_{+}+i \operatorname{Im} B_{+}$and the dimensionless quantity $\omega_{c}$ is given by $\omega_{c}=e B_{0} / m \omega_{p 0}$. Equation (18) describes electrostatic density oscillations driven by electromagnetic (EM) modes, and Eq. (19) describes the influence of density perturbations on the left- and right-hand polarized modes. In order to illustrate the electromagnetic polarizations, we let $N$ $\rightarrow 1$ and consider the linearized version of (19). Integrating the resulting equation once and taking the integration constant as zero, we obtain

$$
\left(\frac{\partial^{2}}{\partial t^{2}}+i \omega_{c} \frac{\partial}{\partial t}+1\right) B_{+}=\left(\frac{\partial}{\partial t}+i \omega_{L}\right)\left(\frac{\partial}{\partial t}-i \omega_{R}\right) B_{+},
$$

where we have introduced the frequency of the left hand mode $\omega_{L} \equiv \sqrt{1+\omega_{c}^{2} / 4}+\omega_{c} / 2$ and of the right hand mode $\omega_{R} \equiv \sqrt{1+\omega_{c}^{2} / 4}-\omega_{c} / 2$. In the absence of electromagnetic fields, $B_{+}=0$, Eq. (18) has a nonlinear solution for $N$

$$
N(t)=\frac{(1+\Delta)}{1+\Delta-\Delta \cos (t)},
$$
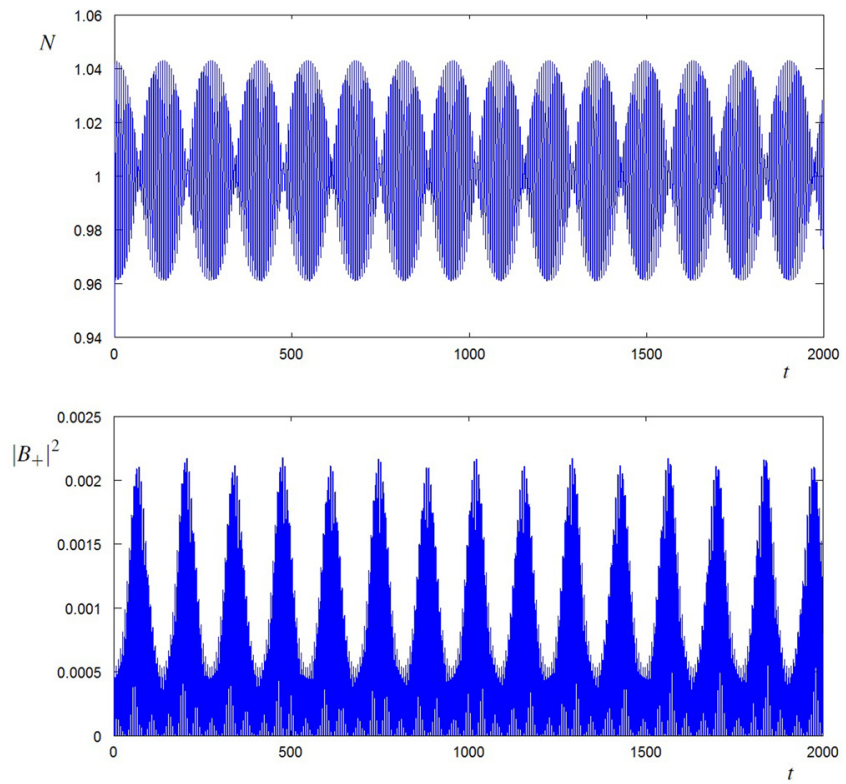

FIG. 1. The density $N$ (upper panel) and the (wave field) magnetic energy density $\left|B_{+}\right|^{2}$ (lower panel) plotted as a function of time for $\omega_{c}=0$. The initial conditions are $N=1.04, d N / d t=0, \quad B_{+}=0, d B_{+} / d t=0.02$, $d^{2} B_{+} / d t^{2}=0$ resulting in linearly polarized electromagnetic fields.

where $\Delta$ is a parameter describing the initial electron density perturbation, or $\Delta=N(0)-1 .^{9}$ Furthermore, linearizing Eq. (18) immediately gives the normalized eigenfrequency $\omega=1$ for the electrostatic mode. The rest of the manuscript is devoted to a numerical study of the full system (18) and (19).

\section{NUMERICAL RESULTS}

Even for fairly modest initial amplitudes $N-1 \sim 0.04$, the nonlinear behavior becomes apparent when the long time
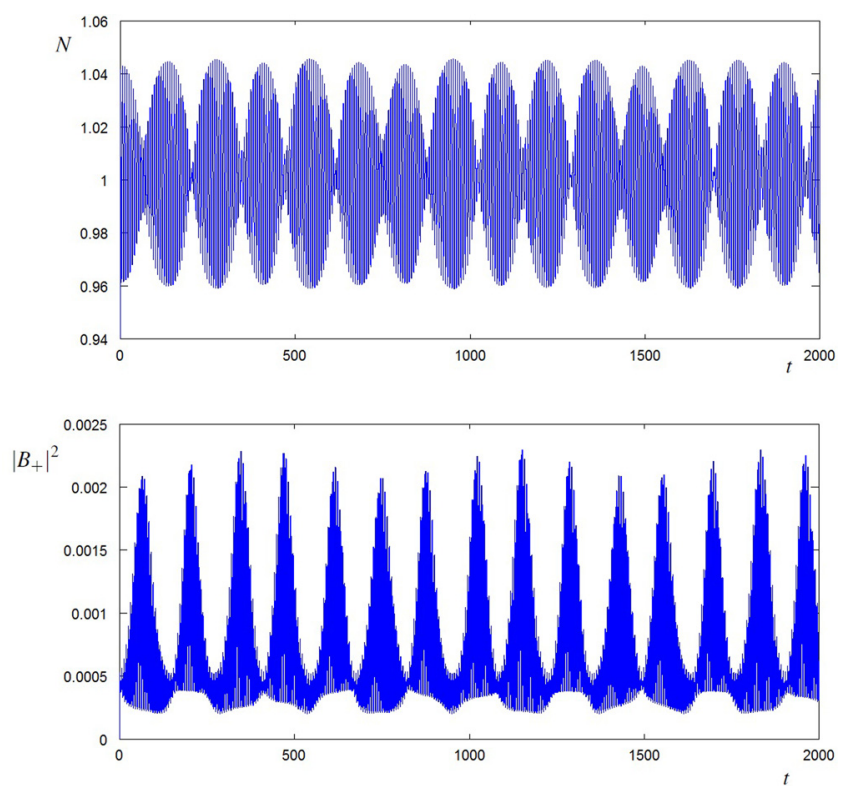

FIG. 2. The density $N$ (upper panel) and the (wave field) magnetic energy density $\left|B_{+}\right|^{2}$ (lower panel) plotted as a function of time for $\omega_{c}=0$. The initial conditions are $N=1.04, d N / d t=0, \quad B_{+}=0, d B_{+} / d t=0.02, d^{2} B_{+} /$ $d t^{2}=0.02 i$ resulting in a time dependent electromagnetic polarization. 

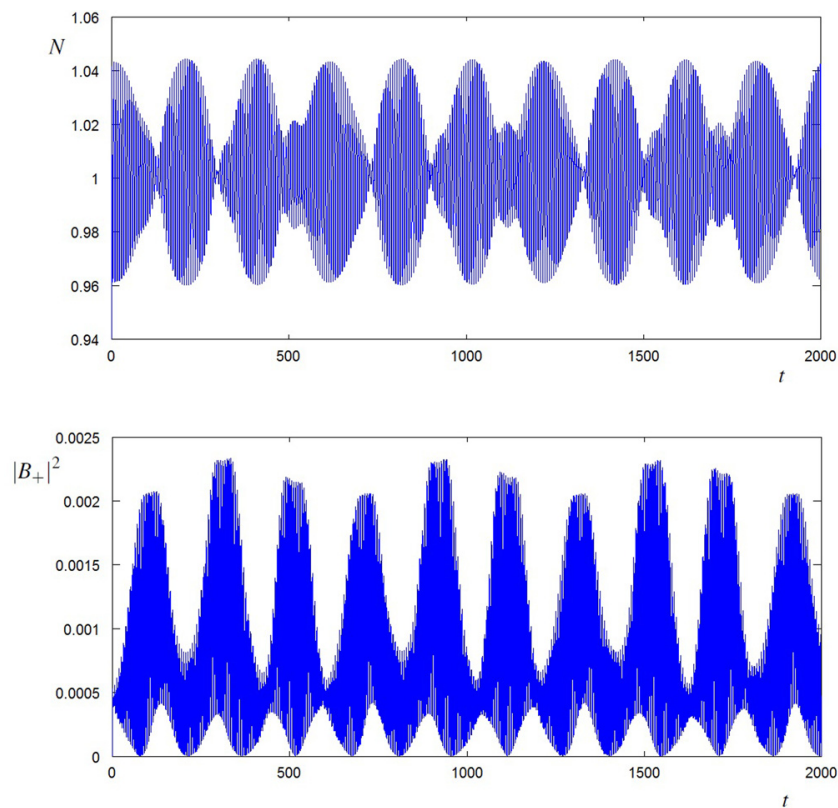

FIG. 3. The density $N$ and the (wave field) magnetic energy density $\left|B_{+}\right|^{2}$ plotted as a function of time for $\omega_{c}=0.05$. The initial conditions are $N=1.04, d N / d t=0, B_{+}=0, d B_{+} / d t=0.02$, and $d^{2} B_{+} / d t^{2}=0.02 i$.
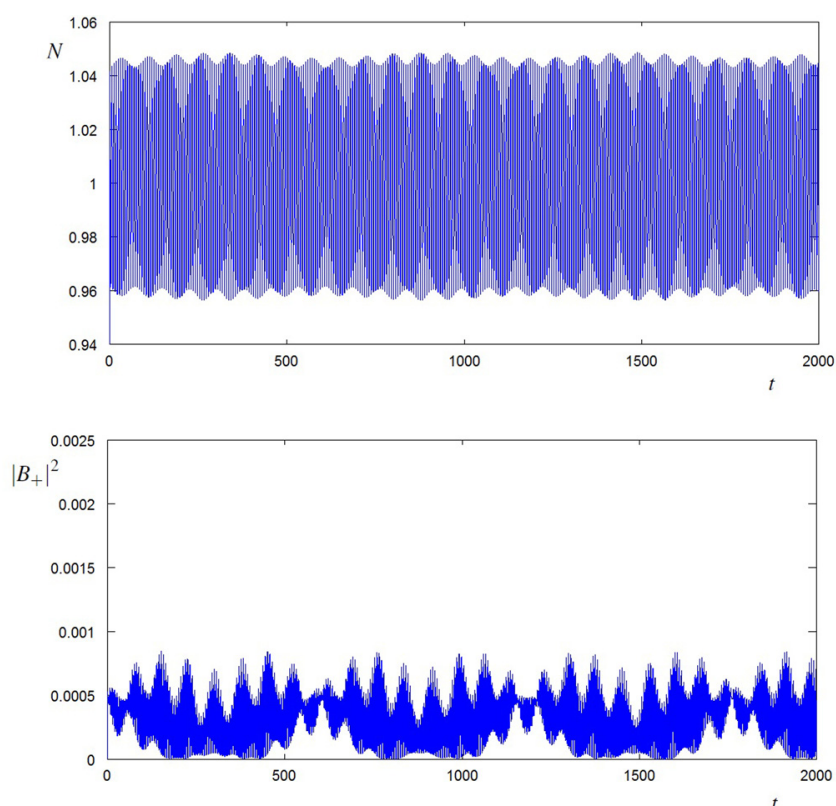

FIG. 4. The density $N$ and the (wave field) magnetic energy density $\left|B_{+}\right|^{2}$ plotted as a function of time for $\omega_{c}=0.2$. The initial conditions are $N=1.04, d N / d t=0, B_{+}=0, d B_{+} / d t=0.02$, and $d^{2} B_{+} / d t^{2}=0.02 i$.

evolution is studied. Starting with the simplest case of no external magnetic field $\left(\omega_{c}=0\right)$ and initial conditions that produce only linearly polarized magnetic fields (i.e., $B_{+}-$ $B_{+}^{*}=0$ for all times), we find that the energy oscillates regularly between electrostatic and electromagnetic degrees of freedom, see Fig. 1. This relative simplicity is dependent on the absence of an external magnetic field that allows linearly polarized EM-modes, but also on initial conditions that keep the polarization fixed. Modifying the initial conditions slightly such that the EM-polarization is allowed to vary as a result of the nonlinear interaction, the amplitude oscillations become significantly more complicated, see Fig. 2. While a quasi-periodic oscillation between electrostatic and electromagnetic modes still can be seen, it is clear that there is much additional structure present. For example, the peaks in the electromagnetic energy are complemented by minima that gradually change shape. The situation is further complicated when the external magnetic field is added. The evolution for the same initial conditions as in Fig. 2 is plotted in Fig. 3, with the difference that the external magnetic field is nonzero, i.e., $\omega_{c}=0.05$. As can be seen, this adds several distinct features to the evolution. For example, the local maxima of the density oscillations now vary in a highly irregular manner. Further increase of the external magnetic field makes the variations in the density oscillations less pronounced (Fig. 4), but the magnetic field evolution is still highly complex.

\section{SUMMARY AND CONCLUSION}

We have here derived Eqs. (18) and (19) that describe the nonlinear interaction between arbitrarily polarized electromagnetic radiation and electrostatic oscillations in a cold magnetized plasma. The ions are considered as immobile, and the electron velocity is non-relativistic, but no other amplitude restrictions have been applied in the derivation. In the absence of an external magnetic field, it is shown that the initial polarization plays an important role for the evolution of the system, and complexity is added since the electrostatic mode can give energy to one electromagnetic polarization at the same time as it gains energy from the other polarization (see Fig. 2). When adding a non-zero external magnetic field into the plasma, the left and right hand polarized modes get different frequencies, which introduces further complexities. This is seen in the structure of the density oscillations (see Fig. 3) and in the magnetic field dynamics (see Fig. 4). A more thorough study of Eqs. (18) and (19) can be a project for further research. Generalizations to include relativistic and/or thermal effects could also be of future interest. Thus, as suggested by the referee, further alternative analytical work may consider a circularly polarized wave magnetic field with constant amplitude and constant density. The resulting nonlinear dispersion relation indicates, then, no nonlinear frequency shift in our nonrelativistic case. However, including relativistic effects (e.g., Refs. 10 and 11) such an approach could lead to interesting effects.

${ }^{1}$ R. C. Davidson, Methods in Nonlinear Plasma Theory (Academic Press, London, 1972).

${ }^{2}$ L. Stenflo, Phys. Scr. 41, 643 (1990).

${ }^{3}$ G. Murtaza and M. Y. Yu, J. Plasma Phys. 57, 835 (1997).

${ }^{4}$ S. Amiranashvili and M. Y. Yu, Phys. Scr. T113, 9 (2004).

${ }^{5}$ A. R. Karimov, J. Plasma Phys. 75, 817 (2009).

${ }^{6}$ G. Lu, Y. Liu, S. Zheng, Y. Wang, W. Yu, and M. Y. Yu, Astrophys. Space Sci. 330, 73 (2010).

${ }^{7}$ C. Maity, A. Sarkar, P. K. Shukla, and N. Chakrabarti, Phys. Rev. Lett. 110, 215002 (2013).

${ }^{8}$ Y. Wang, M. Y. Yu, Z. Y. Chen, and G. Lu, Laser Part. Beams 31, 155 (2013).

${ }^{9}$ G. Brodin and L. Stenflo, Phys. Lett. A 378, 1632 (2014).

${ }^{10}$ L. Stenflo, Phys. Scr. 14, 320 (1976).

${ }^{11}$ P. K. Shukla, N. N. Rao, M. Y. Yu, and N. L. Tsintsadze, Plasma Phys. Rep. 138, 1 (1986). 